\title{
The adequacy of hepatopancreatobiliary training: How does operative exposure and perceived readiness in fellowship translate into subsequent practice?
}

\author{
Jean-Michel Aubin, MD \\ Alexsander K. Bressan, MD \\ Janet P. Edwards, MD \\ Sean C. Grondin, MD \\ Elijah Dixon, MD \\ Rebecca M. Minter, MD \\ D. Rohan Jeyarajah, MD \\ Paul Hansen, MD \\ Amanda B. Cooper, MD \\ Chad G. Ball, MD, MSc
}

Presented at the 12th World Congress of the International Hepato-Pancreato-Biliary Association in Sao Paulo, Brazil in April, 2016.

Accepted Oct. 25, 2016; Early-released Feb. 1, 2017

\section{Correspondence to: \\ C.G. Ball \\ Department of Surgery \\ Foothills Medical Centre \\ University of Calgary \\ Calgary $A B$ T2N $1 \mathrm{~N} 4$ \\ ball.chad@gmail.com}

DOI: $10.1503 /$ cjs. 014216

\begin{abstract}
SUMmaRY
Over the last 3 decades, expansion in the scope and complexity of hepatopancreatobiliary (HPB) surgery has resulted in significant improvements in postoperative outcomes. As a result, the importance of dedicated fellowship training for HPB surgery is now well established, and the definition of formal program requirements has been actively pursued by a collaboration of the 3 distinct accrediting bodies within North America. Although major advances have been made in defining minimum case volume requirements, qualitative assessment of the operative experience remains challenging. Our research collaborative (HPB Manpower and Education Study Group) has previously explored the perceived case volume adequacy of core HPB procedures within fellowship programs. We conducted a 1-year follow-up survey targeting the same cohort to investigate the association between operative case volumes and comfort performing HPB procedures within initial independent practice.
\end{abstract}

T he knowledge currently required for surgical subspecialty practice has clearly exceeded the training capacity of most general surgery residencies. Concomitant expansion of nonsurgical treatments and the increasing complexity of operations have further limited the operative experience of residents and subsequently their confidence to operate independently. The educational impact of resident work hours and autonomy restrictions also remains uncertain. ${ }^{1}$ Most concerning is the lack of confidence to operate independently that has been reaffirmed as a common trait among general surgery graduates seeking postresidency subspecialty training. ${ }^{2}$ In this setting, fellowship programs offer an additional opportunity to improve operative skills, encourage autonomy and build confidence at the subspecialty level before starting independent practice. Improved clinical outcomes for complex operations performed at high-volume centres and growing public demands for safety have further propelled the phenomenon of surgical subspecialization. Currently, more than $80 \%$ of general surgery residents pursue postresidency subspecialty training. ${ }^{3}$

Over the last 3 decades, expansion in the scope and complexity of hepatopancreatobiliary (HPB) surgery has resulted in significant improvements in postoperative outcomes. As a result, the importance of dedicated fellowship training for HPB surgery is now well established, and the definition of formal program requirements has been actively pursued by a collaboration of the 3 distinct accrediting bodies within North America. ${ }^{4}$ Although major advances have been made in defining minimum case volume requirements, qualitative assessment of the operative experience remains challenging. Our research collaborative (HPB Manpower and Education Study Group) has previously explored the perceived case volume adequacy of core HPB procedures within fellowship programs. ${ }^{5}$ This updated 1-year follow-up survey targeted the same 
cohort of fellows from our index study (Fellowship Council-accredited HPB programs in the 2013/2014 academic year), with the objective of understanding the association between operative case volumes and comfort performing HPB procedures within initial independent practice. Survey methodology interrogated 4 domains (demographics, education, practice profile, surgical experience) within 13 core HPB procedures. Our follow-up survey yielded a $91 \%(19 / 21)$ response rate. The mean age of respondents was 36 (range 33-43) years. Respondents had either just completed their first year of practice (11/19), or their second year of fellowship (8/19).

\section{FIRST YEAR OF INDEPENDENT PRACTICE}

All 11 former HPB fellows reported affiliation with an academic institution. Eight are employed in university teaching hospitals (7 with full-time and 1 with part-time academic appointments), and 3 work in community hospitals with a general surgery residency. The average workload exceeds 60 hours per week for most (8/11) and comprises clinical care and surgery (68\% [55\%-90\%]), research (12\% [10\%-25\%]), teaching (9\% [5\%-20\%]), and administrative activities (7\% [5\%-20\%]). All former fellows had a practice mixture beyond isolated HPB surgery: combined with elective general surgery (4/11), acute care surgery (4/11) and liver transplantation (2/11). Reported specialty-specific practice encompassed 39\% liver $(10 \%-50 \%), 39 \%$ pancreas $(20 \%-80 \%)$ and $18 \%$ biliary $(10 \%-30 \%)$ pathologies. Most respondents $(7 / 11)$ were not part of an HPB-specific call schedule, though only 4 participants did not work as part of an HPB group. Dedicated research time involved clinical research (6/11), basic science research (1/11), or both $(4 / 11)$.

\section{Case volumes}

With the exception of open distal pancreatectomies and radical cholecystectomies, average case volumes were lower as an attending surgeon than as a fellow (Table 1). Case volumes for chronic pancreatitis were low during both training and the first year of practice. Hilar cholangiocarcinoma resections, pancreatic necrosectomy, drainage procedures, and celiac plexus blockade also remained infrequent.

\section{Comfort leVel PeRforming HPB Procedures}

Comfort in independently performing core HPB procedures was most often reported for intraoperative ultrasonography (IOUS; 100\%), hepaticojejunostomy (100\%), open $(100 \%)$ and laparoscopic $(94 \%)$ distal pancreatectomy, minor hepatectomy (94\%), pancreaticoduodenectomy (94\%), radical cholecystectomy (87\%), drainage procedures for pancreatic pseudocysts $(87 \%)$ and major hepatectomy (67\%). Presence of a senior HPB surgeon, either as the primary surgeon or assistant, was considered necessary for hilar resection ( $73 \%$ of respondents), celiac plexus blockade $(67 \%)$, and surgery for chronic pancreatitis (47\%; Fig. 1). These comments are very interesting given the first survey observation that fellows often underrate their adequacy of case volumes (major hepatectomies, pancreaticoduodenectomies and laparoscopic distal pancreatectomies) as well as their ability to operate independently (minor hepatectomies, hilar cholangiocarcinomas, celiac plexus blockade) when compared with program directors. ${ }^{5}$ Procedures for chronic pancreatitis deserve special mention. Previously reported low case volumes ${ }^{4}$ remained in this updated survey, and opinions about the comfort-volume association were mixed. This topic

\begin{tabular}{|c|c|c|}
\hline Procedure & Fellow & Staff \\
\hline Major hepatectomy & 46 & 8 \\
\hline Minor hepatectomy & 31 & 15 \\
\hline Pancreaticoduodenectomy & 42 & 12 \\
\hline Open distal pancreatectomy & 5 & 8 \\
\hline Laparoscopic distal pancreatectomy & 15 & 4 \\
\hline Drainage procedure for pancreatic necrosis & 8 & 1 \\
\hline Surgery for chronic pancreatitis & 2 & 2 \\
\hline Pancreatic necrosectomy & 8 & 1 \\
\hline Radical cholecystectomy & 5 & 6 \\
\hline Hepaticojejunostomy & 21 & 3 \\
\hline Resection of hilar cholangiocarcinoma & 11 & 1 \\
\hline Celiac plexus block & $<1$ & $<1$ \\
\hline IOUS & 144 & 27 \\
\hline Complex biliary reconstruction & 6 & 4 \\
\hline
\end{tabular}




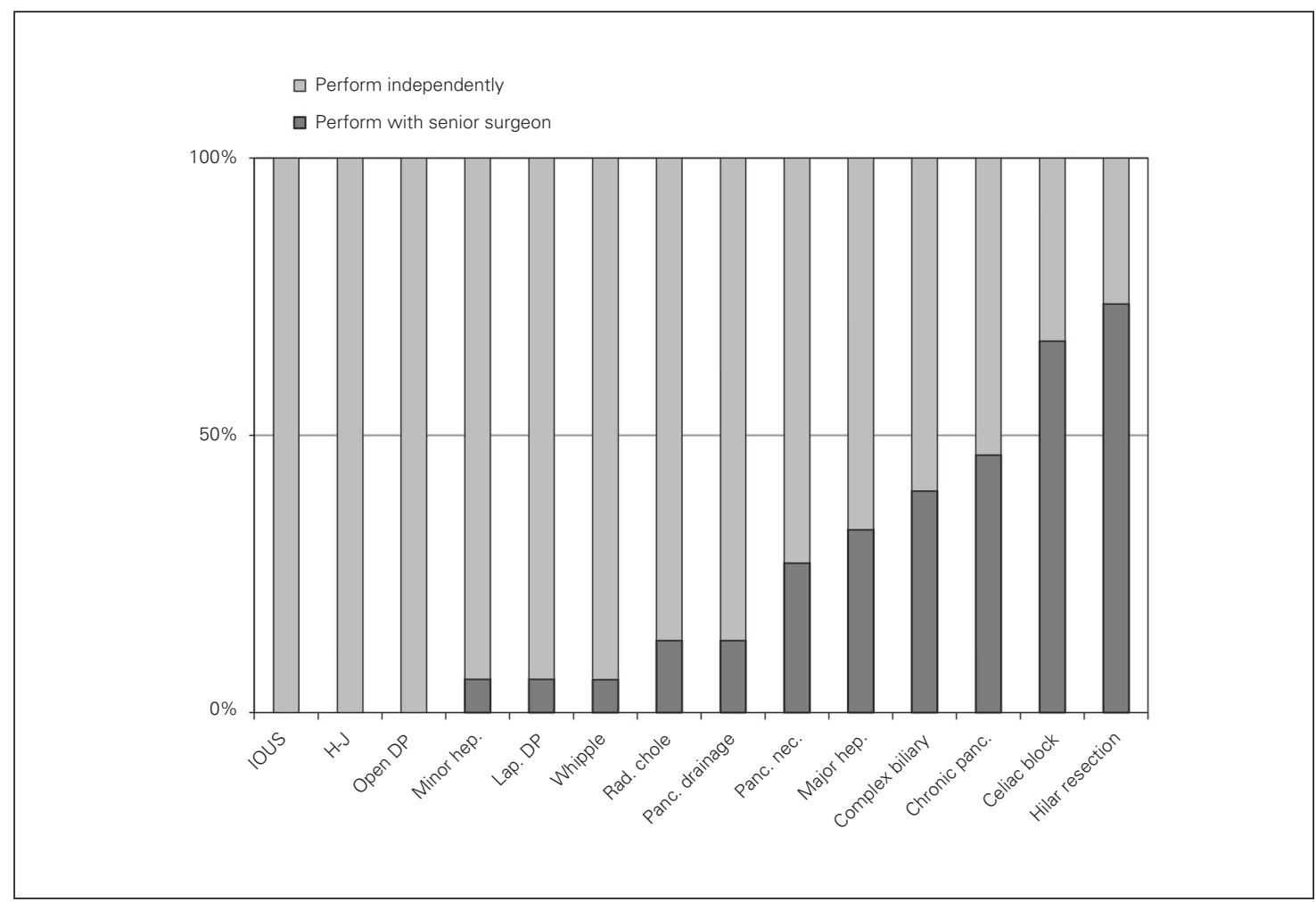

Fig. 1. Comfort level performing critical steps of core hepatopancreatobiliary (HPB) procedures during the first year of independent practice. DP = distal pancreatectomy; $\mathrm{H}-\mathrm{J}=$ hepatico-jejunostomy; IOUS = intraoperative ultrasonography.

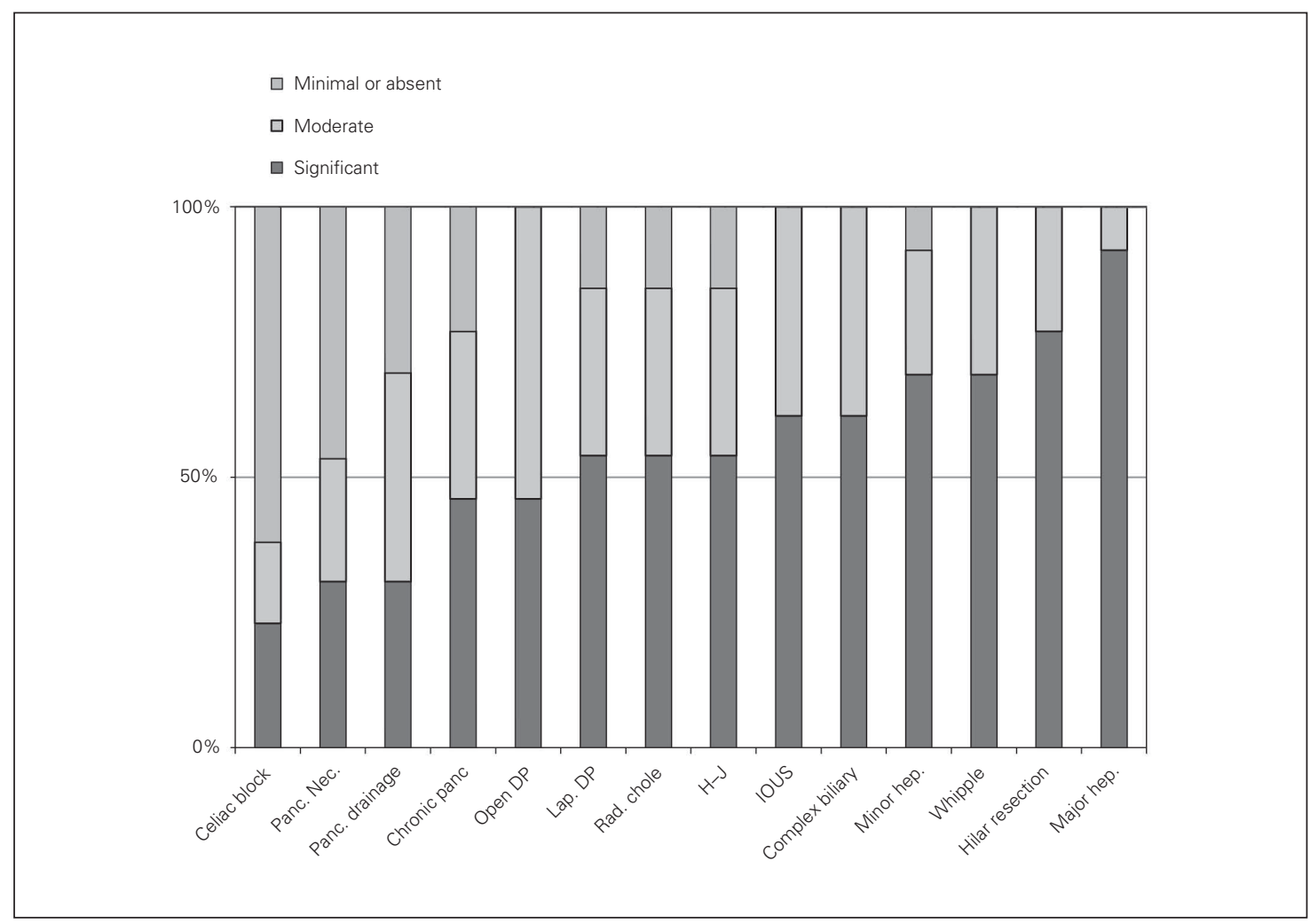

Fig. 2. Perceived impact of case volumes during fellowship training on comfort performing core hepatopancreatobiliary (HPB) procedures during the first year of independent practice. DP = distal pancreatectomy; $\mathrm{H}-\mathrm{J}=$ hepaticojejunostomy; IOUS = intraoperative ultrasonography. 
remains a challenge for HPB educators, given that sound surgical judgment for pancreatitis can be extremely complex, more challenging than pancreatic oncology, and often reliant on high-volume exposure as well as a profound understanding of the disease. Laparoscopic distal pancreatectomies displayed a low $(<50 \%)$ reported rate of "good or excellent" case volume, but a high degree of preparedness during fellowship training. This updated survey confirms a high degree of comfort performing laparoscopic distal pancreatectomies, with $80 \%$ of respondents performing them independently and an additional 13\% feeling comfortable enough to teach it to junior residents. These findings do not follow the trends identified for all other procedures and therefore reflect a relative degree of perceived technical simplicity. This is likely a direct result of mastery of the open procedure with a subsequent extrapolation to the laparoscopic methodology. It is also interesting to note that the structured exposure to IOUS in recent years within the FC/AHPBA-accredited fellowship programs (including curricular requirements via an IOUS course) has improved both case volumes and the experience of graduating fellows (high use and comfort by junior staff).

\section{VOLUME-COMFORT ASSOCIATION}

Most respondents (83\%) believed case volume during training significantly affected their operative comfort level during initial independent practice. This volume-comfort association was most often reported for major hepatectomy (92\%), hilar resection (77\%), pancreaticoduodenectomy (69\%) and minor hepatectomy (69\%; Fig. 2). For less frequently performed procedures (celiac plexus blockade and pancreatitis interventions), operative volume during training was not perceived to significantly impact comfort. Hilar lesions deserve additional comment given that less than $40 \%$ of fellows reported "good or excellent" exposure in our previous study. Because resectable hilar lesions are relatively rare malignancies, gaining high-volume exposure is unlikely to occur across all training programs. Despite the reality that much can be learned and transposed from other operations, such as major hepatectomies, hilar dissections and complex biliary reconstructions, surgical educators must continue to develop alternative approaches to teaching these complex procedures.

\section{OTHER CONSIDERATIONS}

The number of HPB fellowship programs in North America was perceived to be excessive by $57 \%$ of respondents. All respondents believed they were adequately prepared during fellowship training, and half felt their goals were "completely" achieved: clinical expertise (100\%), technical expertise (93\%), development of a professional network (64\%) and improved employability (43\%). After the first year of practice, $42 \%$ were "very satisfied," and $50 \%$ were "somewhat satisfied" in their jobs. While joining a group, half reported no issues with the transition, but adjustment to group practice was described as an obstacle not anticipated during training. Other hurdles encountered when entering practice included the logistics of billing and of setting up a practice. Suggested improvements in HPB fellowship training included greater autonomy while operating (suggested by $86 \%$ ), discrete rotations at high-volume centres for specific diseases (64\%) and a formal examination at the end of the fellowship (64\%). In contrast to general surgery residency training, respondents reported a low interest $(14 \%)$ in operative simulation to address areas of low preparedness and comfort.

\section{Conclusion}

Our updated survey reaffirms the importance of case volumes in developing fellows' confidence across a wide range of operative procedures encompassed by HPB surgery. Appropriate confidence to operate independently, successful placement of former fellows in academic HPB positions and overall perception of accomplishment of training goals are reassuring findings that support ongoing efforts to elevate the quality of HPB fellowship programs within North America.

Affiliations: From the University of Calgary, Calgary, Alta. (Aubin, Bressan, Edwards, Grondin, Dixon, Ball); the University of Texas Southwestern Medical Centre, Dallas, TX (Minter); the Methodist Dallas Medical Center, Dallas, TX (Jeyarajah); the Providence Medical Center, Portland, OR (Hansen); and the Penn State Hershey Medical Center, Hershey, PA (Cooper).

Competing interests: None declared.

Contributors: All authors contributed substantially to the conception, writing and revision of this article and approved the final version for publication.

\section{References}

1. Grover BT, Kothari SN. Fellowship training: need and contributions. Surg Clin North Am 2016;96:47-57.

2. Klingensmith ME, Cogbill TH, Luchette F, et al. Factors influencing the decision of surgery residency graduates to pursue general surgery practice versus fellowship. Ann Surg 2015;262:44955, discussion 54-5.

3. Fowler DL, Hogle NJ. The Fellowship Council: a decade of impact on surgical training. Surg Endosc 2013;27:3548-54.

4. Jeyarajah DR, Berman RS, Doyle M, et al. Consensus Conference on North American Training in Hepatopancreaticobiliary Surgery: a review of the conference and presentation of consensus statements. Ann Surg Oncol 2016;16:1086-93.

5. Bressan AK, Edwards JP, Grondin SC, et al. The adequacy of hepato-pancreato-biliary training: How closely do perceptions of fellows and programme directors align? HPB (Oxford) 2015;17:791-5. 